\title{
Impacto en la comunidad del sector no estatal. Caso de estudio restaurante Nirvana
}

Impact on the non-state sector community. Nirvana restaurant case study

Julia Ma. Espinosa Manugás. ${ }^{1}$, Beatriz Romaní Bendig. ${ }^{2}$ \& Leonardo Angulo García ${ }^{3}$

\begin{abstract}
The position that the non-state sector has acquired in recent years in the capital, with an emphasis on gastronomic activity, both in the structure of competition and in its capacity to generate value, justifies the conduct of this research, which has as objective, to assess the impact of the restaurant "Nirvana" in the community, for which a questionnaire was used in the form of a survey prepared for the purposes of this study and validated by the Alpha coefficient of Crombach. The number of respondents was defined based on the total number of families that live in the blocks closest to the restaurant, using the sample carculator program. The results were processed through parametric statistics, factor analysis and the Chi square contingency test, using the SPSS version 22 program. It was found that the restaurant positively impacts the community, basically due to its contribution to economic activity, variable highly correlated with the generation of employment, living conditions and personal income of the inhabitants: Similarly, it was observed that the perception of the impact of the restaurant by the respondents shows a significant difference ( $\mathrm{p}<0.05$ ), being better valued for those born in the locality, those who reside in it for a longer period of time, adults over 60 years of age, women and those who live closer to it.
\end{abstract}

Key Words: Community, Restaurant, Impact, Restoration.

\section{Resumen}

El posicionamiento que ha ido adquiriendo en los últimos años el sector no estatal en la capital, con énfasis en la actividad gastronómica, tanto en la estructura de la competencia como en su capacidad generadora de valor, justifica la realización de la presente investigación, que tiene como objetivo, valorar el impacto del restaurante "Nirvana" en la comunidad, para lo cual se empleó un cuestionario en forma de encuesta elaborada a

\footnotetext{
${ }^{1}$ Dr. C. Facultad de Turismo. Universidad de La Habana. julia_espinosa@ftur.uh.cu

${ }^{2}$ MSc. Facultad de Turismo. Universidad de La Habana. bea_romani@ftur.uh.cu

${ }^{3}$ Lic. Leonardo Angulo García. Inmobiliaria del turismo. Mintur. leoangulo@gmail.com
} 
los fines del presente estudio y validada por el coeficiente Alfa de Crombach. La cantidad de encuestados se definió a partir del total de núcleos familiares que habitan en las cuadras más cercanas al restaurante, empleando para ello el programa Sample size carculator. Los resultados se procesaron a través de la estadística paramétrica, el análisis factorial y la prueba de contingencias de Chi cuadrado, empleando el programa SPSS versión 22. Se constató que el restaurante impacta positivamente en la comunidad, básicamente por su contribución a la actividad económica, variable altamente correlacionada con la generación de empleo, condiciones de vida e ingresos personales de los pobladores: De igual modo se observó que la percepción del impacto del restaurante por los encuestados presenta diferencia significativa $(\mathrm{p}<0,05)$, siendo valorada de mejor manera por los nacidos en la localidad, los que residen en esta por un mayor periodo de tiempo, los adultos mayores de 60 años, las femeninas y los que habitan más próximo a esta.

Palabras Clave: Comunidad, Restaurante, Impacto, Restauración.

\section{Introducción}

En los últimos años como resultado de los cambios que se han realizado a partir de la actualización del Modelo Económico Cubano, se ha promovido entre otros aspectos la ampliación del trabajo en el sector no estatal, con el fin de obtener una gestión más eficiente de la economía cubana.

En consecuencia, se ha ido configurando una sociedad más multiespacial y multiactoral desde el punto de vista socio productivo, en la cual se ha consolidado la presencia cada vez mayor de actores privados (Izquierdo y Morin, 2017).

Este incremento sustancial experimentado por el sector no estatal ha tenido de manera general un comportamiento positivo en el entorno en que se desarrolla, originando una nueva fuente generadora de empleo a la población, competencias entre negocios y entidades estatales ya existentes, obligando a la innovación de estas empresas y la necesidad de crear un producto nuevo y diferente (Triana y Maqueira, 2016).

Destaca dentro de esta forma de gestión, el posicionamiento que ha ido adquiriendo la restauración privada, especialmente en la capital, creando responsabilidades tales como el cuidado de la higiene pública y la promoción de la cocina cubana como elemento de identidad cultual de la nación, beneficiando tanto a los empleadores y empleados, como a la localidad en que se encuentran inmersos y a la sociedad cubana en su conjunto

Esta forma de trabajo ha generado una competencia en la prestación de servicios cuyo impacto no solo se ve en los resultados económicos y financieros, sino en las estrategias de gestión a partir de la política comercial que se pone en práctica, lo que limita a los restaurantes regidos por la administración estatal a adoptar nuevas formas de estrategias y diversidad de sus productos (Vidal y Pérez, 2012 y Domenech, 2018).

Teniendo en cuenta lo antes expuesto se concibió el presente trabajo el cual tiene como objetivo valorar el impacto del restaurante "Nirvana" en la comunidad, investigación que 
permite a partir del análisis de los resultados obtenidos establecer acciones para eliminar los elementos que inciden de manera negativa en la localidad y potenciar los aspectos positivos, mejorando así la relación entre el restaurante, el entorno en que se encuentra y sus habitantes.

\section{Desarrollo}

\section{Metodología empleada}

Para dar cumplimiento al objetivo, inicialmente se recopiló información sobre los límites de la circunscripción donde se encuentra enclavado el restaurante, obteniendo que la misma se compone de 4 manzanas y 12 cuadras, de estas últimas, seis son las más cercanas al restaurante, de ahí que fueron las consideradas en el estudio.

A través de la consulta del libro central de electores de la circunscripción se determinó el total de núcleos familiares de las cuadras seleccionadas (537), y a partir de dicha información y mediante el programa Sample size calculator, para un nivel de confianza del $90 \%$ y un error muestra del $10 \%$, se determinó el tamaño de la muestra, esto es los núcleos familiares a los cuales se le iba a solicitar la información requerida para conocer el impacto del restaurante en la comunidad, obteniendo un valor de 113.

La información se recopiló a través de una encuesta, empleando un cuestionario elaborado por los autores, conformado por cuatro preguntas cerradas y una abierta.

Se indagó el efecto del restaurante en la comunidad, desde el punto de vista económico, recreativo, cultural, como generador de empleo e incidencia en las condiciones de vida de los pobladores, empleando una escala de Likert de tres categorías que varía de mucho a nada. De igual forma se valoró la influencia de la instalación en el comportamiento social e ingresos personales y familiares de los habitantes, y su responsabilidad en cuanto a la higiene y preservación del medio ambiente, mejora de la infraestructura y conservación del entorno, utilizando una escala de Likert de 5 categorías (1 muy positiva, 2 positiva, 3 sin consecuencias, 4 negativa, 5 muy negativa). Se solicitó a los encuestados que expusieran sus datos personales (edad, sexo, escolaridad, ocupación y tiempo de residencia en la localidad.

Se estimó la confiabilidad del cuestionario por el coeficiente alfa de Cronbach, índice de consistencia que toma valores entre 0 y 1 y que es aceptable cuando es mayor de 0,7 (Hernández, Fernández, Baptista, 2010), y la concordancia de las respuestas dadas por los encuestados, mediante el análisis factorial, técnica que permite una reducción de la dimensionalidad de los datos, a partir de la correlación entre las variables. Se confirmó la correlación mediante la prueba de esfericidad de Bartlett y la pertinencia del análisis a través del estadístico Kaiser-Meyer-Olkin (KMO), el cual indica que es factible cuando es superior a 0,7 (Lloret, Ferrer, Hernández y Tomás, 2014). Para conocer si las variaciones en las respuestas pudieran estar condicionas por las características personales 
de los encuestados y la cercanía de sus residencias al restaurante, se realizó la prueba de contingencia de Chi cuadrado para un nivel de confianza del $95 \%$.

\section{Resultados}

Al comprobar la fiabilidad del cuestionario se obtuvo un coeficiente Alfa de Cronbach de 0,912 , lo que indica que la encuesta aplicada es confiable y los resultados son válidos.

La tabla 1 muestra los porcentajes obtenidos para los indicadores socio demográficos de los encuestados.

Tabla 1. Datos socio-demográficos de los encuestados

\begin{tabular}{|c|c|c|}
\hline \multicolumn{2}{|c|}{ Indicadores } & $\%$ \\
\hline \multirow{3}{*}{ Autóctono del Municipio } & $\mathrm{Si}$ & 71,1 \\
\hline & No & 28,9 \\
\hline & $18-30$ & 31,6 \\
\hline \multirow[t]{2}{*}{ Edad } & $30-60$ & 46,1 \\
\hline & Más de 60 & 22,3 \\
\hline \multirow{2}{*}{ Sexo } & Masculino & 57,9 \\
\hline & Femenino & 42,1 \\
\hline \multirow{4}{*}{ Grado de escolaridad } & Secundaria & 15,8 \\
\hline & Preuniversitario & 61,9 \\
\hline & Técnico medio & 46,1 \\
\hline & Profesional & 21,1 \\
\hline \multirow{5}{*}{ Ocupación } & Cuentapropista & 21,1 \\
\hline & Obrero & 25,0 \\
\hline & Desempleado & 13,1 \\
\hline & Jubilado & 15,8 \\
\hline & Estudiante & 3,9 \\
\hline \multirow{4}{*}{ Tiempo de residencia en la comunid } & ene-20 & 23,7 \\
\hline & & \\
\hline & $21-40$ & 44,7 \\
\hline & Más de 40 & 31,6 \\
\hline
\end{tabular}

Fuente: Elaboración propia

Como puede observarse, el 71,1\% de la población encuestada es nacida en el municipio, lo que constituye un elemento positivo a la hora de obtener opiniones y juicios sobre los más diversos aspectos del entorno, incluyendo los negocios no estatales. Predomina el rango de edad de 30 a 60 años $(46,1 \%)$, y el sexo masculino $(57,9 \%)$. 
Con relación al grado de escolaridad se evidencia que solo el 22,4\% de los encuestados es universitario, frente a una mayoría, 61,9\% que posee doce grados (preuniversitario y técnico medio).

Las ocupaciones más frecuentes en la población encuestada es la de obreros en el sector estatal, para un 25\% del total, seguido de los profesionales. El 21,1\% manifestó ser cuentapropista sin especificar la labor que realizan. El 76,3\% reside en la comunidad por más de 20 años, lo que evidencia el conocimiento que tienen de la misma, su sentido de pertenencia y responsabilidad social.

La figura 1 refleja las respuestas obtenidas para las variables: actividad económica, recreación, intercambio cultural, generación de empleo y condiciones de vida-entorno en la misma.

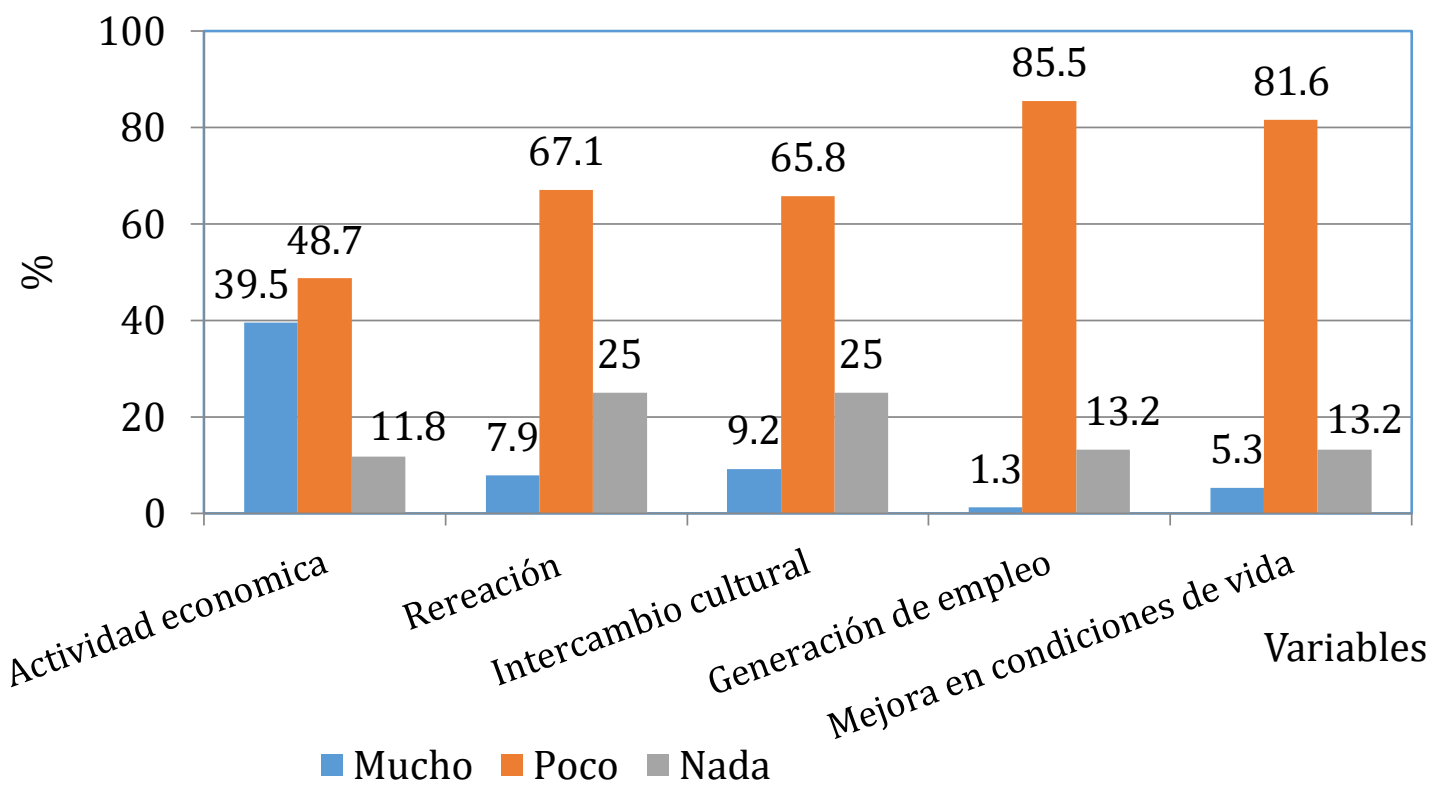

Figura 1. Incidencia del restaurante para cinco de las variables analizadas

Fuente: Elaboración propia

Como queda demostrado, un $48,7 \%$ de la población coincide en que el aporte del restaurante a la actividad económica de la comunidad es poco y un $11,8 \%$ plantea que es nula, sin embargo, el 39,5\% opina lo contrario, al considerar que contribuye mucho y que el restaurante tiene un impacto relativamente positivo en la población. Esos porcentajes, aunque reflejan cierto equilibrio entre una y otra respuesta, representan opiniones divididas que pueden estar condicionada por las características individuales de los encuestados.

El comportamiento anterior es similar al del resto de las variables analizadas, En todos los casos la mayoría opina que el restaurante contribuye poco a la comunidad en lo relativo a la recreación, el intercambio cultural, mejora de las condiciones de vida del entorno y la generación de empleo, siendo esta última la variable más devaluada. 
La Tabla 2 hace referencia a la influencia del restaurante en la comunidad, teniendo en cuenta los indicadores de comportamiento social y los ingresos personales. Se evidencia un comportamiento satisfactorio para ambas variables.

Tabla 2. Influencia del restaurante en el comportamiento social e ingresos de la comunidad

\begin{tabular}{|c|c|}
\hline Respuestas & $\%$ \\
\hline \multicolumn{2}{|c|}{ Comportamiento social } \\
\hline Muy positivo & 51,3 \\
\hline Positivo & 30,3 \\
\hline Sin consecuencias & 13,2 \\
\hline Negativo & 3,9 \\
\hline \multicolumn{2}{|c|}{ Ingresos personales } \\
\hline Muy negativo & 1,3 \\
\hline Muy positivo & 1,3 \\
\hline Positivo & 73,7 \\
\hline Sin consecuencias & 14,5 \\
\hline Negativo & 7,9 \\
\hline Muy negativo & 2,6 \\
\hline
\end{tabular}

Fuente: Elaboración propia

El 81,6\% de los encuestados aportó opiniones positivas respecto a la influencia del restaurante en el comportamiento social y de ese porcentaje la mayor parte $(51.3 \%)$ consideró dicha influencia "Muy positiva". Tal resultado confirma que el flujo de turistas en la zona no es fuente de disturbios, como tampoco lo es en el interior del restaurante, a pesar de estar enclavado en una barriada declarada de alta peligrosidad. Sobre el indicador referido a los ingresos personales, el 75,0\% lo considera "positivo" o "muy positivo", siendo este un resultado favorable para la comunidad desde el punto de vista económico. La figura 2 exhibe los resultados relacionados con la responsabilidad que asume el restaurante frente al medio ambiente, la infraestructura y conservación del entorno. 


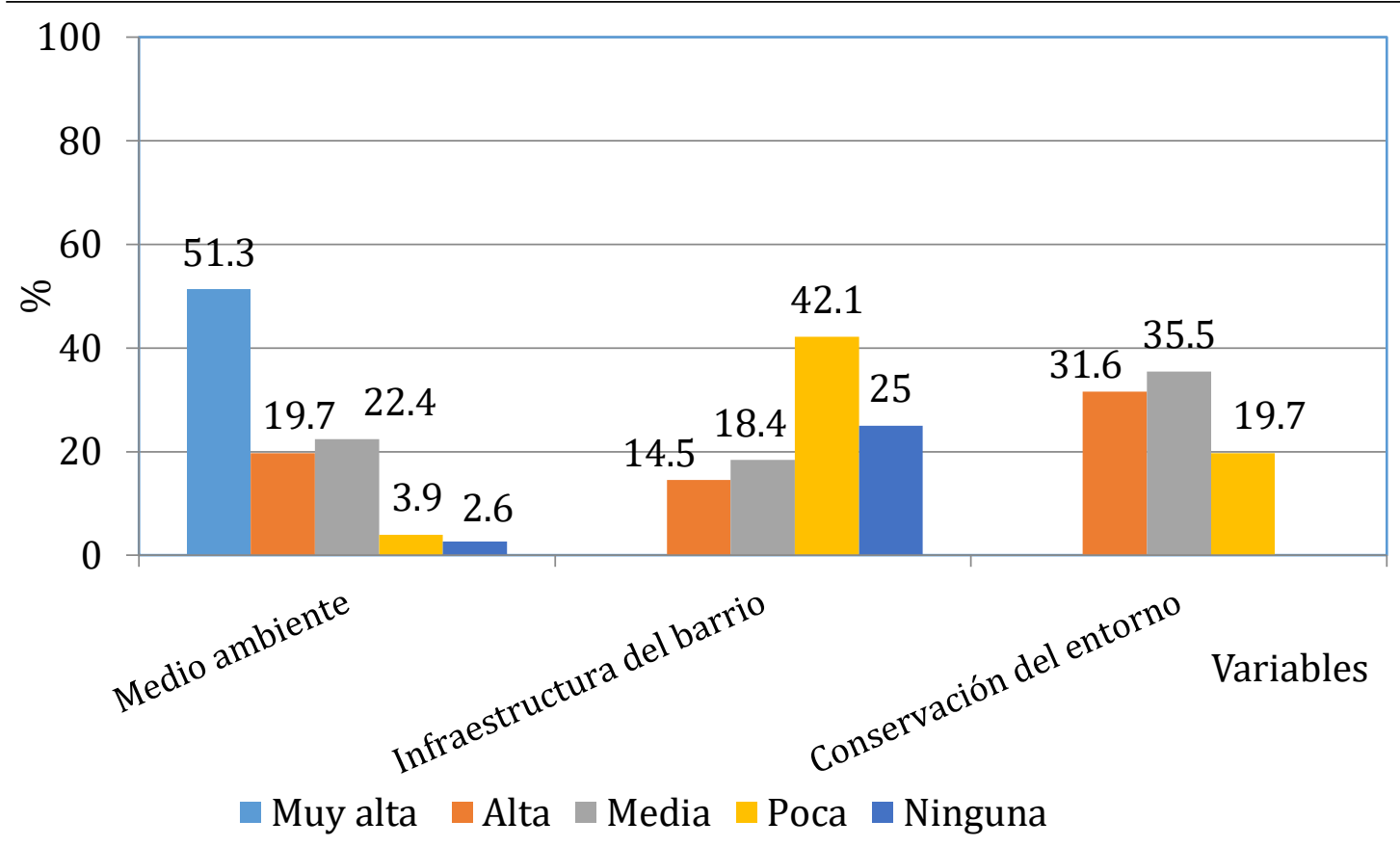

Figura 2. Incidencia del restaurante en relación al medio ambiente, la infraestructura y conservación del entorno.

Fuente: Elaboración propia

Se aprecia que para el $71 \%$ de los pobladores el restaurante contribuye de manera favorable a la preservación del medio ambiente, los que consideran que la preocupación y gestión por la eliminación de desechos sólidos y líquidos es elevada, y se materializa a través de las coordinaciones realizadas por el propietario del restaurante con instancias estatales encargadas de la higiene pública.

Con respecto a la incidencia del restaurante en la mejora de la infraestructura de la zona, los encuestados en su mayoría $(67,1 \%)$ estiman que es poca o ninguna, siendo la variable más devaluada. Durante la realización de la encuesta, algunos manifestaron que las obras constructivas y de restauración solo se ponen en marcha en el inmueble donde se encuentra ubicado el restaurante y no se tiene en cuenta el entorno a pesar de que sería conveniente mejorar su imagen.

El resultado anterior guarda relación con el encontrado al evaluar la variable “conservación del entorno", donde el 55,2\% la evaluó de poca o ninguna, los argumentos emitidos fueron los expuestos anteriormente en cuanto a que el restaurante se enfoca a la mejora de su instalación y no a su entorno. Algunos encuestados plantearon que no consideran que esta variable sea responsabilidad total del restaurante, ya que existen instancias estatales a las que les corresponde el mantenimiento de la infraestructura pública,

En la tabla 3 se exhiben los resultados de la prueba de Kaiser-Meyer-Olkin. Se evidencia que es pertinente realizar el análisis factorial, por cuanto $\mathrm{KMO}=0,828$, mayor de $0,70 \mathrm{y}$ 
la prueba de esfericidad de Bartlett confirma la correlación existente entre las variables analizadas, al obtener un valor Chi-cuadrado de 703,261 para una significación $(\mathrm{p}<0,05)$.

Tabla 3. Resultado de la Prueba de KMO

\begin{tabular}{|c|c|c|}
\hline Medida Kaiser-Meyer-Olkin de a & ción de muestreo & ,828 \\
\hline Prueba de esfericidad de Bartlett & Aprox. Chi-cuadrado & 703,261 \\
\hline & $\mathrm{gl}$ & 55 \\
\hline & Sig. &, 000 \\
\hline
\end{tabular}

Fuente: Programa SPSS. Versión 22

Las mayores correlaciones se obtuvieron para las variables que se reportan en la tabla 4, constatándose un resultado congruente.

El impacto del restaurante en la actividad económica de la comunidad guarda estrecha vinculación con la generación de empleo, las condiciones de vida de sus habitantes y sus ingresos personales. Existe una relación significativa entre las actividades de recreación que se promueven en la comunidad y el intercambio cultural entre sus habitantes; el comportamiento social de estos se encuentra en correspondencia con el respeto del medio ambiente, y las acciones que impacta en el mejoramiento de la infraestructura del barrio, lógicamente contribuye a la conservación del entorno

Tabla 4. Variables más correlacionadas

\begin{tabular}{llll}
\hline \multicolumn{2}{c}{ Variables } & \multicolumn{2}{c}{ Coeficiente de correlación } \\
\hline & Generación de empleo & 0,909 & \\
\cline { 2 - 3 } Actividad económica & Condiciones de vida- & 0,880 & \multirow{2}{*}{0,786} \\
& entorno & 0,911 & \\
\cline { 2 - 3 } & Ingresos personales & 0,759 & \multirow{2}{*}{0,791} \\
\cline { 2 - 3 } & Generación de empleo & \\
\hline Recreación & Intercambio cultural & 0,997 \\
\hline Comportamiento social & Respeto al ambiento & 0,712 \\
\hline Infraestructura del barrio & Conservación del entorno. & 0,852 \\
\hline
\end{tabular}

Fuente: Elaboración propia

El análisis de los resultados obtenidos evidencia que existe variabilidad en las opiniones de los encuestados en cuanto al impacto que ha tenido el restaurante en la comunidad, lo que fue corroborado mediante el análisis factorial, el cual mostró que las respuestas pueden agruparse en dos conglomerados distantes entre sí (Figura 3). Estas discrepancias pueden explicarse a partir de la información derivada de la prueba de contingencia que se presenta en las tablas 5 y 6 . 


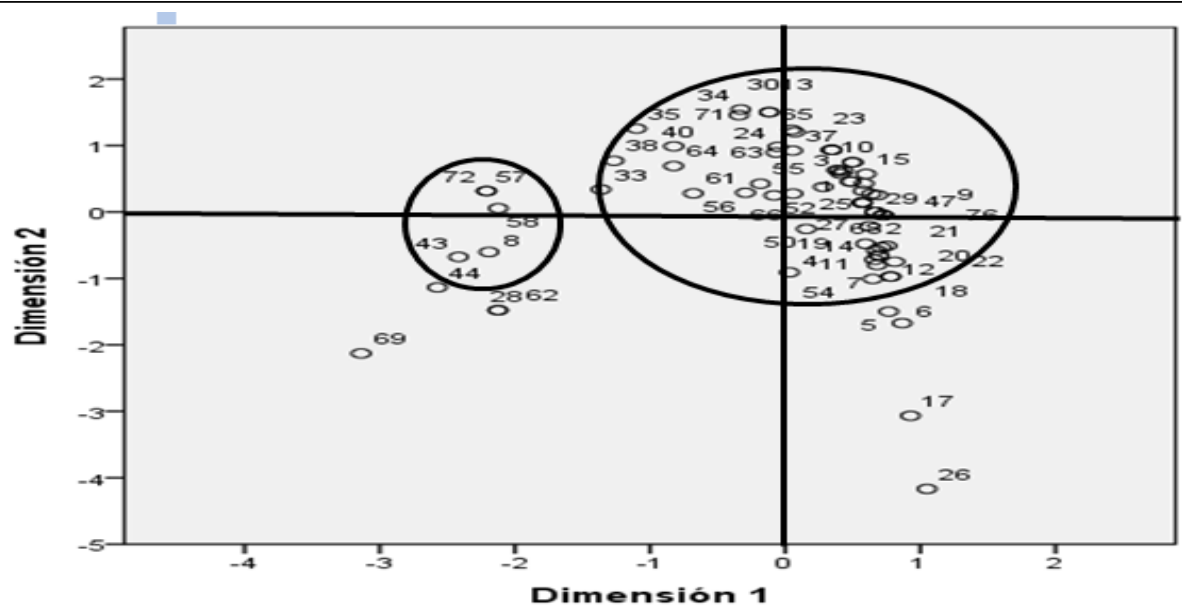

Figura 3. Resultados del análisis factorial

Fuente: Programa SPSS. Versión 22

Como se observa en la tabla 5, existe diferencia significativa $(\mathrm{p}<0,05)$ en la respuesta de los encuestados para la mayoría de las variables analizadas, en función de si son autóctonos del municipio, la edad, el sexo y el tiempo de residencia en la localidad y para el $60 \%$ y $20 \%$ de estas en el caso del grado de escolaridad y la ocupación de los residentes respectivamente.

Tabla 5. Resultados de la prueba de contingencia de Chi cuadrado (según características de los encuestados)

\begin{tabular}{lcccccc}
\hline \multicolumn{1}{c}{ Variables } & \multicolumn{5}{c}{ Indicadores } \\
\cline { 2 - 7 } & $\begin{array}{c}\text { Autóctono } \\
\text { del } \\
\text { municipio }\end{array}$ & Edad & Sexo & Escolaridad & Ocupación & $\begin{array}{c}\text { Tiempo de } \\
\text { residencial }\end{array}$ \\
\hline Actividad económica & $0,15 *$ & $0.11 *$ & $0,09 *$ & $0,03 \mathrm{~ns}$ & $0,001 \mathrm{~ns}$ & $0,03 \mathrm{~ns}$ \\
Recreación & $0,67 *$ & $0,18 *$ & $0,27 *$ & $0,05 *$ & $0,000 \mathrm{~ns}$ & $0,37 *$ \\
Intercambio cultural & $0,56 *$ & $0.61 *$ & $0,23 *$ & $0,06 *$ & $0,000 \mathrm{~ns}$ & $0,75 *$ \\
Generación de empleo & $0,81 *$ & $0,16 *$ & $0,17 *$ & $0,21 *$ & $0,01 \mathrm{~ns}$ & $0,13 *$ \\
Condiciones de vida-entorno & $0,10 *$ & $0,22 *$ & $0,09 *$ & $0,014 \mathrm{~ns}$ & $0,005 \mathrm{~ns}$ & $0,25 *$ \\
Comportamiento social & $0,32 *$ & $0,81 *$ & $0,88 *$ & $0,01 \mathrm{~ns}$ & $0,57 *$ & $0,72 *$ \\
Ingresos & $0,70 *$ & $0,19 *$ & $0,31 *$ & $0,16 *$ & $0,001 \mathrm{~ns}$ & $0,22 *$ \\
Medio ambiente & $0,21 *$ & $0,36 *$ & $0,60 *$ & $0,006 \mathrm{~ns}$ & $0,06 *$ & $0,16 *$ \\
Infraestructura & $0,78 *$ & $0,04 \mathrm{~ns}$ & $0,72 *$ & $0,10 *$ & $0,001 \mathrm{~ns}$ & $0,45 *$ \\
Conservación del entorno & $0,95 *$ & $0,35 *$ & $0,32 *$ & $0,42 *$ & $0,005 \mathrm{~ns}$ & $0,60 *$ \\
\hline
\end{tabular}

$\square$ : Diferencia significativa para $\mathrm{p}<0,05 \quad$ ns: No significativa

Fuente: Elaboración propia 
Respecto a la variable autóctono del municipio, se pudo observar que las respuestas más favorables se evidenciaron en los sujetos que son nacidos en la localidad. Esto se traduce, en que esta parte de la población puede comparar la situación antes y después de apertura del restaurante, y tener una opinión más amplia del impacto que este ha generado. Lo mismo se aplica a la variable tiempo de residencia donde se evidencia mayoría de respuestas positivas en los que llevan más de 40 años residiendo en el lugar.

Se observó que los mayores de 60 años se mostraron más positivos al evaluar el impacto del restaurante en la comunidad. Se constató también que para todos los indicadores estudiados las mayores respuestas positivas fueron emitidas por las féminas, lo que puede atribuirse a que por las características propias del negocio de restauración y los complementarios que se han originado a partir de la apertura del restaurante los empleos que se han generado han sido básicamente para mujeres.

En la tabla 6 se aprecia variación significativa $(\mathrm{p}<0,05)$ para el $60 \%$ de las variables, en función de la cercanía al restaurante de la vivienda de los encuestados. Se advierte que dicha diferencia se origina básicamente en las variables que guardan una mayor relación con la situación económica y la calidad de vida de la comunidad, obteniéndose los mejores resultados en la cuadra donde se encuentra enclavado el restaurante.

Tabla 6. Resultados de la prueba de contingencia de Chi cuadrado (según cercanía de las viviendas de los encuestados al restaurante)

\begin{tabular}{lc}
\hline Variables & Significación \\
\hline Actividad económica & $0,50 *$ \\
Recreación & $0,03 \mathrm{~ns}$ \\
Intercambio cultural & $0,04 \mathrm{~ns}$ \\
Generación de empleo & $0,33 *$ \\
Condiciones de vida-entorno & $0,09 *$ \\
Comportamiento social & $0,40 *$ \\
Ingresos personales y familiares & $0,50 *$ \\
Medio ambiente & $0,10 *$ \\
Infraestructura & $0,000 \mathrm{~ns}$ \\
Conservación del entorno & $0,000 \mathrm{~ns}$ \\
\hline
\end{tabular}

Fuente: Elaboración propia

\section{Conclusiones}

- La apertura del restaurante objeto de estudio ha incidido de manera favorable en la comunidad del entorno, aunque no de igual manera para todas las variables analizadas en la presente investigación. Destaca su influencia en el mejoramiento 
de las condiciones económicas de sus pobladores, variable altamente correlacionada con la generación de empleo, condiciones de vida e ingresos personales de los pobladores.

- La percepción del impacto del restaurante por los pobladores de la comunidad es heterogénea y presenta diferencia significativa $(\mathrm{p}<0,05)$, siendo valorada de mejor manera por los nacidos en la localidad, los que residen en esta por un mayor periodo de tiempo, los adultos mayores de 60 años, las femeninas y los que habitan más próximo a esta.

\section{Referencias bibliográficas}

Domenech, H. (2018). Propuesta de estrategias para aumentar la competitividad del barrestaurante Floridita. Tesis en opción al título de Master en Gestión Turística. Facultad de Turismo. Universidad de La Habana, La Habana, Cuba.

Hernández, R., Fernández, C. y Baptista P. M. (2010). Metodología de la investigación. D.F: The McGraw-Hill.

Izquierdo, O. y Morín, J. (2017). El modelo económico y social de desarrollo socialista y los actores laborales no estatales. La participación laboral y el sistema político en el contexto de la actualización del Sistema Económico y Social cubano. En O. Izquierdo, y H. Burchardt (comps.) Trabajo docente y sociedad. Cuba bajo la óptica de los estudios socio laborales. La Habana: Editorial UH.

Lloret, S., Ferrer, A., Hernández, A. y Tomás, I. (2014). El análisis factorial exploratorio de los ítems: una guía práctica, revisada y actualizada. Universidad de Murcia. España. Anales de Psicología, 30(3), 1151-1169.

Triana, C. J. y Maqueira, J. (2017). El sector no estatal desde la perspectiva institucional. En M. Bergara y V. Hidalgo (coords.) Transformaciones económicas en Cuba: Una perspectiva institucional. La Habana: Anales de la Academia de Ciencias de Cuba

Vidal, P. y Pérez, O. (2012) Apertura al cuentapropismo y la microempresa, una pieza clave del ajuste estructural. En P. Vidal y O. Pérez, (comps.) Miradas a la economía cubana. El proceso de actualización. La Habana: Editorial Caminos.

\section{Ciencia
Digital
$\varepsilon^{d \text { thoul }^{\prime}}$}




\section{PARA CITAR EL ARTÍCULO INDEXADO.}

Espinosa Manugás, J. M., Romaní Bendig, B., \& Angulo García, L. (2021). Impacto en la comunidad del sector no estatal. Caso de estudio restaurante Nirvana. Explorador Digital, 5(1), 173-184. https://doi.org/10.33262/exploradordigital.v5i1.1496

\section{\Ciencia}

El artículo que se publica es de exclusiva responsabilidad de los autores y no necesariamente reflejan el pensamiento de la Revista Explorador Digital.

El artículo queda en propiedad de la revista y, por tanto, su publicación parcial y/o total en otro medio tiene que ser autorizado por el director de la Revista Explorador Digital.
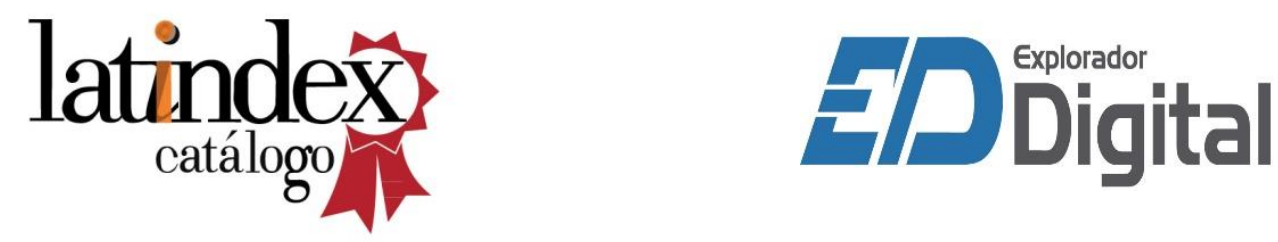